\title{
El uso de las TIC digitales por parte del personal docente y su adecuación a los modelos vigentes
}

\author{
The Use of Digital ICT by Teachers and Their Adaptation to Current Models
}

O uso de TIC digital por professores e sua adaptação aos modelos atuais

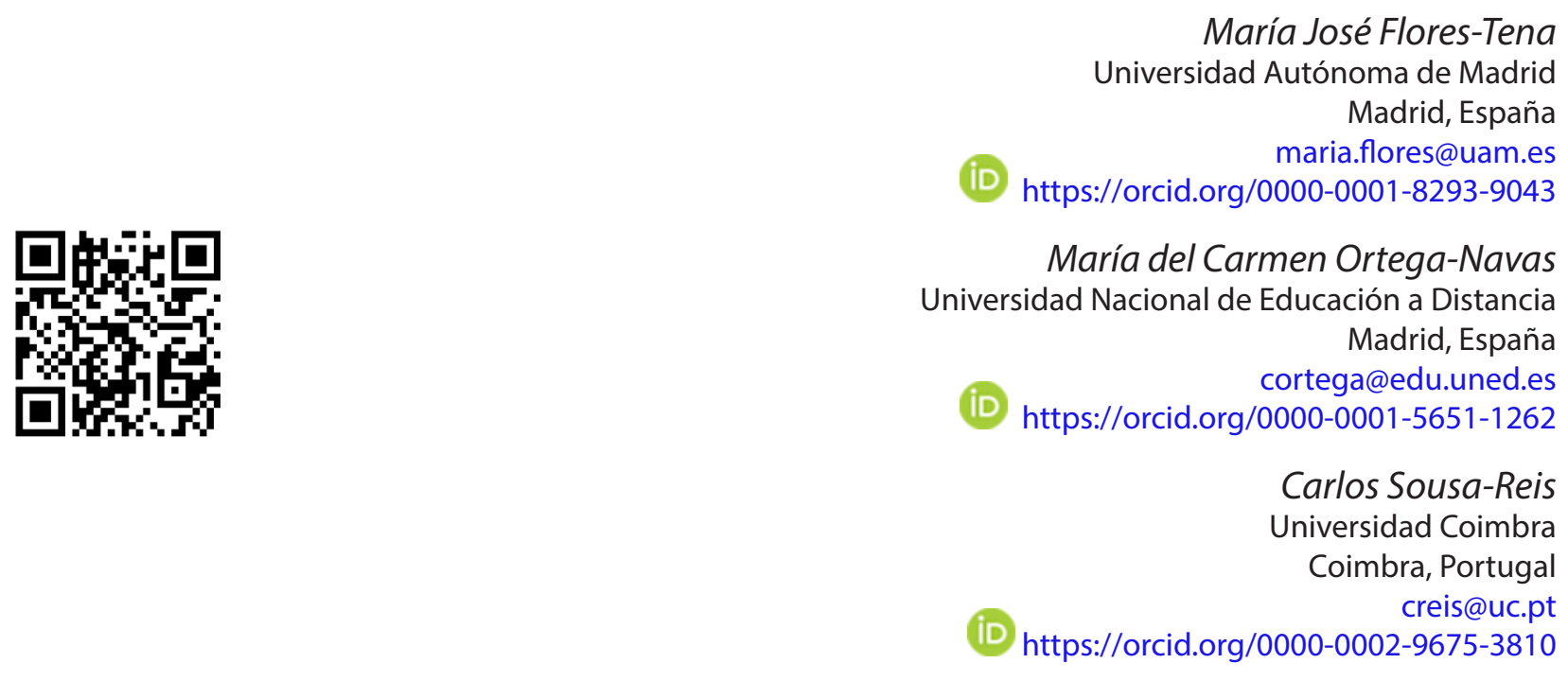

Recibido • Received • Recebido: 20 / 06 / 2019

Corregido • Revised • Revisado: 26 / 10 / 2020

Aceptado • Accepted • Aprovado: 04 / 12 / 2020

\begin{abstract}
Resumen: En el artículo presentamos un estudio en el que se pretende constatar la importancia y adecuación sobre el uso de las tecnologías de la información y de la comunicación (TIC) en el ámbito escolar para su mayor adecuación a la realidad. Esta cuestión queda plasmada en el objetivo general, centrado en determinar el uso que hace el personal docente de las tecnologías digitales, su adecuación a los modelos vigentes en las aulas y su repercusión en el proceso de enseñanza-aprendizaje de sus estudiantes, teniendo en cuenta las debilidades presentes en los fundamentos pedagógicos esgrimidos por algunos de los más recientes estudios sobre el uso y optimización de las TIC digitales en el aula. El estudio se enmarca en un diseño de corte cuantitativo, no experimental, descriptivo y correlacional. En el estudio participaron 100 docentes de Educación Secundaria Obligatoria (ESO) en institutos de Coímbra (Portugal). Se elaboró un cuestionario creado ad hoc para conocer el uso y frecuencia de las nuevas tecnologías en el aula por parte del personal docente. Los datos recogidos en los cuestionarios fueron analizados a través del paquete estadístico Statistical Package for the Social Sciences. Las principales conclusiones confirman que es necesario aún un debate profundo sobre los fundamentos epistemológicos implícitos en el uso de las nuevas herramientas digitales; sobre
\end{abstract}


http://doi.org/10.15359/ree.25-1.16

http://www.una.ac.cr/educare

educare@una.ac.cr

si las TIC son una herramienta docente adecuada, acorde a los modelos educativos del presente, en términos tiempo-coste para un mejor rendimiento y éxito académico del estudiantado. De hecho, el debate está más vivo que nunca, las nuevas tecnologías se han insertado, cada vez más, en el ámbito educativo, durante el COVID-19, lo cual ha implicado, incluso, la suspensión de actividades docentes presenciales que han conllevado el uso de las nuevas tecnologías en el aula de forma inmediata.

Palabras claves: Calidad educativa; epistemología; estrategias pedagógicas; informática educativa; modelos pedagógicos; tecnologías de la información y la comunicación.

Abstract: In this article, we present a study intended to verify the importance and adequacy of the use of information and communication technologies (ICT) in the school environment for their greater adaptation to reality. This issue is reflected in the general objective, focused on determining the use made by teaching staff of new technologies in classrooms and their impact on the teaching-learning process of their students, taking into account the weaknesses present in the pedagogical foundations put forward by some of the most recent studies on the use and optimization of digital ICT in the classroom. The study has a quantitative, non-experimental, descriptive, and correlational design. In the study, 100 teachers of Compulsory Secondary Education (ESO) in institutes of Coimbra (Portugal) participated. An put forward questionnaire was developed to determine the use and frequency of new technologies in the classroom by the teaching staff. The data collected in the questionnaires were analyzed using the Statistical Package for the Social Sciences. The main conclusions confirm that there is still a need for an in-depth debate on the epistemological foundations implicit in the use of the new digital tools, and on whether ICTs are an adequate teaching tool, according to the current educational models, in time-cost terms for better student achievement and academic success. In fact, the debate is more than ever alive; new technologies have been increasingly implemented in the educational field during COVID-19 pandemics. This situation has even implied the suspension of inperson teaching activities that have led to the use of new technologies in the classroom immediately.

Keywords: Educational quality; epistemology; pedagogical strategies; Educational informatics; pedagogical models, information and communication technologies.

Resumo: Apresentamos nesse artigo um estudo no qual se pretende verificar a importância e a adequação do uso das tecnologias de informação e comunicação (TIC) no ambiente escolar para a sua maior adaptação à realidade. Esta questão está refletida no objetivo geral da mesma, centrada em determinar a utilização que o corpo docente faz das novas tecnologias em sala de aula e o seu impacto no processo de ensino-aprendizagem dos estudantes, considerando as fragilidades presentes nas bases pedagógicas de alguns dos estudos mais recentes sobre a utilização e otimização das TICs digitais na sala de aula. O estudo está enquadrado em um desenho quantitativo, não experimental, descritivo e correlacional. No estudo, 100 professores do Ensino Secundário Obrigatório (ESO) participaram em institutos de Coimbra (Portugal). Foi elaborado um questionário criado ad hoc para conhecer a utilização e frequência das novas tecnologias em sala de aula pelo corpo docente. Os dados coletados nos questionários foram analisados por meio do sistema estatístico Statistical Package for the Social Sciences. As principais conclusões confirmam que ainda é necessário um debate aprofundado sobre os fundamentos epistemológicos implícitos na utilização das novas ferramentas digitais, questionando se as TIC são um instrumento de ensino 


\begin{abstract}
adequado, de acordo com os modelos educacionais da atualidade, em termos de tempo-custo, para um melhor desempenho dos estudantes e sucesso acadêmico. Na verdade, o debate está mais atual que nunca, as novas tecnologias foram cada vez mais inseridas no campo educacional, durante o COVID-19, o que implicou, inclusive, a suspensão das atividades de ensino presencial, que levaram ao uso imediato de novas tecnologias na sala de aula.
\end{abstract}

Palavras-chave: Qualidade educacional; epistemologia; estratégias pedagógicas; informática educacional; modelos pedagógicos, tecnologias de informação e comunicação.

\title{
Introducción y estado de la cuestión
}

El proceso integral de enseñanza-aprendizaje puede concretarse al momento en el que se produce una interacción simbólica efectiva entre un sujeto emisor (docente) y un sujeto receptor (estudiante) mediante un código lingüístico y cultural.

Más aún, conviene puntualizar que dicha interacción, docente-estudiante en el proceso pedagógico no necesariamente implica, que lo que deba asimilar el sujeto receptor tiene que ser una copia fiel del mensaje inicialmente emitido; más bien tendríamos una continua traducción de significados.

Numerosas contribuciones sostienen que la educación ha invertido en nuevas tecnologías para lograr una mejor calidad educativa en el sistema educativo, así como que enseñar al alumnado en el uso de las nuevas tecnologías permite el aprendizaje y aplicación del conocimiento en las aulas y en la vida diaria, conforme a la sociedad en la que vivimos de un modo más eficiente (Área, 2005; Area y Guarro, 2012; Bernete, 2010; Bustos y Román 2011; Cabero Almenara, 2007; Cornejo y Tapia, 2011; García Aretio, 2009; Hodges et al. 2020; León Martínez y Tapia Rangel, 2013). Otras señalan que las tecnologías contribuyen a la reflexión que debe hacer el personal docente para enfrentarse a la sociedad cambiante en todos los aspectos, fruto de la globalización (Cornejo y Tapia, 2011; Fainholc, 2011; Fernández Enguita, 2020; Ortega-Navas, 2011).

A lo largo de este estudio se ha constatado la necesidad de renovación del sistema educativo para hacer frente a unas necesidades socioeducativas latentes en el marco escolar actual que definen un sistema obsoleto y con falta de adecuación a los nuevos retos de la realidad educativa. Entre las diferentes visiones de los agentes educativos, se puede apreciar una percepción general de atraso del sistema educativo frente a la creciente evolución de la sociedad y, con ello, constatar la necesidad de adecuación del ámbito escolar en aspectos como las nuevas tecnologías y la renovación de su marco normativo para su mayor adecuación a la realidad.

El objetivo general de este estudio se ha centrado en determinar el uso de las TIC digitales por parte del personal docente y su adecuación a los modelos pedagógicos vigentes. 
http://doi.org/10.15359/ree.25-1.16

http://www.una.ac.cr/educare

educare@una.ac.cr

Para finalizar, se ha tratado de poner en relieve la necesidad de incorporación de profesionales, que enseñen o puedan enseñar cualquier disciplina haciendo uso de las nuevas tecnologías o de las TIC, al marco escolar, para la mejora de la acción educativa a nivel personal, social y colectivo. Sin embargo, la falta de conocimiento en el proceso o enseñanza aprendizaje virtual hace que sea necesaria la formación para docentes y estudiantes en el manejo de las nuevas tecnologías o de las TIC.

Los cambios que se han venido produciendo tras la irrupción de las nuevas tecnologías de la información y la comunicación digital han abierto un nuevo abanico en las oportunidades y demandas en la educación, caracterizados, según a Flores-Tena y Ortega-Navas (2018), por su simultaneidad, instantaneidad, interactividad, asincronía y ubicuidad. Así, se comprende que el debate académico actual se centre en los aspectos relacionados con la implementación, optimización y evaluación del uso de las TIC en el aula.

Lo cierto es que la revolución hipermediática contemporánea está llevando a extremos inimaginables las propias condiciones comunicacionales y transformando radicalmente las formas en que se produce gran parte de las relaciones sociales interpersonales. De modo que resulta aún más desconcertante que ya ni siquiera sea significativo que la interacción simbólica requiera una concreción material y temporal, pudiendo llegar a ser exclusivamente virtual (Bernete, 2010).

Cabe destacar que los medios tecnólógicos imponen retos insospechados de difícil manejo y conducción. En parte, porque si los medios comienzan por diluirse, luego se relativizan y encuentran su lugar complementario, dividiendo espacios y funciones en el complejo sistema que pasan a formar (Cruz, 2002). La era digital ha supuesto una habilidad de destreza educativa para desenvolverse en los entornos digitales (Eshet-Alkalai,2004).García-Peñalvoy Seoane-Pardo (2015) hacen referencia a diferentes temas relacionados con las comunidades de aprendizaje, donde los sujetos participantes se implican en las mismas metas y en los mismos objetivos. McLuhan (2013) demostró que todo nuevo medio tecnológico extiende las capacidades comunicativas del ser humano, lo cual no significa que semejante proceso vuelva obsoleto parte del sistema de medios existente, condenándole a la desaparición. Véase el caso de cómo la escritura dio por terminada, en su momento, la era del lenguaje oral, así como tampoco la aparición de la imprenta acabó con la comunicación escrita, ni la radio desterró la imprenta, o la televisión arruinó el cine y ni el multifuncional smartphone desplazó los medios existentes, sino más bien los integró. Así llegamos al punto de destacar que la anterior observación constituye una premisa crucial con vistas a los propósitos planteados en el presente análisis.

Igualmente es más evidente que, desde la revolución que ha supuesto la irrupción de los medios digitales, las TIC están desmaterializando el ámbito de las relaciones sociales y desmontado el andamiaje conceptual de los elementos presentes en la comunicación. Así, al 
http://doi.org/10.15359/ree.25-1.16

mismo tiempo que se han venido desdibujando los roles tradicionalmente asignados al ente emisor y al ente receptor en el proceso de comunicación, ya no está tan claramente definido, incluso, a quién le corresponde producir el mensaje. Una situación que, en parte, estudios como los de Cairncross (1997), Fujita et al. (1999) y Quah (1996), definen como la muerte de las distancias geográficas. Y dado que, de esta forma, se pone en duda cuál es el estímulo y cuál es la reacción al establecerse un enlace comunicacional, la incertidumbre llega hasta el extremo de cuestionarse cuál es el momento en que se está produciendo -de facto- el mensaje mismo. Además, pese a todas estas paradojas, lo que resulta realmente desconcertante es la cuestión de saber dónde se encuentra el mensaje al momento de establecerse el enlace comunicacional.

Ahora bien, aunque las TIC digitales están contribuyendo al cambio en las formas y circunstancias en las que se está dando la comunicación en las relaciones interpersonales en la sociedad y, en particular, dentro del aula, tal y como señalan Bernete García (2014) y Marqués Graells (2013), la cuestión es en qué medida, en qué grado de eficiencia y hasta qué punto es deseable dicha transformación en el proceso de enseñanza-aprendizaje. Sin perder de vista que los nuevos medios no tienen por qué extinguir todos los anteriores, más aún, es necesario no olvidar que la prodigalidad de medios ha traído dos problemas: 1. adecuar los medios a la educación, dándole prioridad sobre cuestiones tal vez más antiguas, pero aun así más importantes; 2. producir los contenidos adecuados a la educación, presionando para que se produzcan y se introduzcan, juntamente con los medios, en las escuelas, de modo rápido e inexorable (Kaplún, 2001).

\section{Las TIC y su enfoque didáctico}

La segunda mitad del siglo XX llevó a la sociedad a un nuevo proceso transformador radical. Estas transformaciones en particular, tal y como lo estableció McLuhan (2013), han estado marcadas por los cambios profundos que se han ido sucediendo en los sistemas de comunicación; sistemas que la sociedad misma genera para facilitar sus interrelaciones. Los cambios en la sociedad debidos a la irrupción de los TIC digitales son ya palpables y están siendo ampliamente debatidos (Bernete, 2010 y Marqués Graells, 2013).

El problema radica en que dicha inexorabilidad hace que el debate de su implementación en el aula sea visto, preponderantemente, desde su necesaria adecuación y no desde su adecuada necesidad. Dicho de otro modo, la discusión sobre la irrupción de las TIC en el aula implica abordar cuestiones fundamentales que aún no han sido resueltas en el ámbito educativo (Altamirano Herrera y Lera Mejía, 2017; Bernete García, 2014).

Por otra parte, el tema del uso de las TIC en la sociedad viene siendo, incluso, uno de los temas más relevantes en diferentes ámbitos. Por ejemplo, las Naciones Unidas, Comisión de Ciencia y Tecnología para el Desarrollo (2014), emitió este mensaje: 
Las políticas inclusivas en materia de TIC para el desarrollo deben abordar las cuestiones de la asequibilidad, la accesibilidad, la idoneidad del contenido y la educación para velar por que las TIC contribuyan a reducir las desigualdades y a promover la inclusión social y económica. (p. 17)

En este mismo sentido, y casi, con las mismas palabras y las mismas elusiones, tal como indica el Banco Interamericano de Desarrollo (citado por Bernete, 2014): "Las [TIC] tienen un gran potencial para apoyar el desarrollo de mejores aprendizajes y son un factor clave para cerrar las brechas, integrando lo diverso y dando acceso a la información y al conocimiento" ( p. 73).

Al respecto, la reflexión académica sobre el uso de las TIC en el aula se sigue circunscribiendo, en gran medida, a esta forma de enfocar el tema. La incorporación de las tecnologías de la información y la comunicación en las aulas de los centros educativos es normalmente prescrita como "una medida necesaria y urgente que llevará sin duda a una mejora significativa en el proceso de aprendizaje y enseñanza" (Altamirano Herrera y Lera Mejía, 2017, p. 416).

Conviene puntualizar que se excluyen del presente análisis los planteamientos que, como el de Marqués Graells (2013), limitan sus posiciones a la necesidad de desarrollar un adecuado adiestramiento operativo de las TIC digitales, sin considerar otros aspectos como su pertinencia y relevancia. Este mismo autor habla de un proceso de alfabetización digital al que equipara al de enseñar a leer y a escribir; es decir, el manejo operativo de estas herramientas resulta un medio didáctico más, necesario para acometer los procesos de enseñanza y aprendizaje.

Retomando, el objeto de análisis que aquí se plantea es la justificación del uso de las TIC en el aula como herramienta didáctica en sí misma, lo cual se fundamenta, en gran medida, desde planteamientos como que estas "abren una nueva forma de acceder a la información y son un gran puente de comunicación para el contacto y el aprendizaje" (Tello Díaz Maroto y Cascales Martínez, 2015, p. 355). Con este argumento de base, los autores infieren, además, que su uso "permite mejorar la calidad educativa, fomentando la Justicia Social" (Tello Díaz Maroto y Cascales Martínez, 2015, p. 357).

Este posicionamiento de partida lleva a estos mismos autores, incluso, a denostar cualquier otro posible enfoque. Llegan a señalar que "toda la alfabetización digital universal, con sus diversas metodologías de utilización en el aula ... debe ser justificada mucho más desde el punto de vista de la inclusión social que desde la perspectiva pedagógica" (Tello Díaz Maroto y Cascales Martínez, 2015, pp. 361-362).

Yaún desdeñando aquella visión simplista de lo novedoso como valor positivo en sí mismo -fenómeno que caracterizó al proceso transformador sociocultural surgido tras la revolución industrial y hasta finales del siglo XX-se llega a afirmar, sin mayor sustentación, que la adopción de las TIC va encaminada al mayor logro del aprendizaje, aumentando además de la motivación 
http://doi.org/10.15359/ree.25-1.16

http://www.una.ac.cr/educare educare@una.ac.cr

del alumnado la participación de la comunidad en las escuelas y generando contextos donde la convivencia sea positiva (McCharen et al., 2011).

Partiendo de tal enfoque, estos mismos autores concluyen que es necesario integrar los elementos digitales al sistema educativo para seguir transmitiendo el conocimiento con nuevas herramientas hasta reducir el uso de los libros de texto en el aula, mediante la innovación en las aulas como estrategia para la mejora de la calidad educativa en términos de aprendizaje y motivación del estudiantado (McCharen et al., 2011).

Lo más delicado de este planteamiento no es el objetivo mismo, sino el hecho de que ignora el postulado esencial de McLuhan (2013), quien señaló que la adopción de una nueva herramienta comunicacional no implicaba, en absoluto, el destierro de las existentes, sino la extensión de las capacidades ya desplegadas.

Bernete (2010) es enfático al señalar que el hecho de que puedan constatarse muchas de las ventajas de utilizar las TIC, su mera utilización no supone que sea siempre igualmente provechosa para todas las personas; y ni siquiera implica, por sí misma, una mejor o peor calidad del aprendizaje.

Cabe destacar que a semejanza de muchos otros casos, lo que el uso de la tecnología requiere es análisis y regulación, sin que tengamos que considerarnos seres apocalípticos tecnológicos, sea al respecto de su general uso en la sociedad, o lo que en el ámbito pedagógico se refiere.

En primer lugar, hay que poner en tela de juicio el propio determinismo tecnológico implícito en tales posicionamientos. No hay duda de que las transformaciones sociales y culturales introducidas por la evolución de los medios es una dinámica importante; sin embargo, hay que dosificarla con la referencia a los contextos, a los contenidos y a las intenciones, pues es determinante conocer la naturaleza, alcance y preponderancia -política o psicológica- de sus consecuencias (Briggs y Burke, 2002). De ahí que solo haciendo referencia a los contextos se podrá ver claramente la naturaleza del fenómeno que queremos estudiar.

En muchos casos, los medios parecen ser verdaderos catalizadores de los cambios sociales, más que sus causantes. Tal como la educación, la "tecnología requiere y al mismo tiempo produce cambios sociales y organizacionales" (Briggs y Burke, 2002, p. 214).

En segundo lugar, hay que notar que el determinismo tecnológico elude un debate más profundo, y aún más urgente, si cabe, sobre los aspectos deontológicos que, tras la irrupción de las TIC en la sociedad y, consecuentemente, en las estrategias desplegadas en el aula. Siguiendo a Díaz-Barriga (2008), señala que las TIC aplicadas en educación no garantizan ni la inclusión, ni la equidad social, tampoco la calidad o la innovación; en numerosas ocasiones la tecnología produce modelos de enseñanza tradicionales, y es necesario crear nuevas teorías de diseño 
http://doi.org/10.15359/ree.25-1.16

http://www.una.ac.cr/educare

educare@una.ac.cr

educativo con el uso de tecnologías que vayan de acuerdo con las necesidades actuales, a fin de lograr conocimientos útiles para la resolución de problemas relevantes y con sentido social.

Tampoco debemos olvidar que los cambios en la sociedad de nuestro país, y que no hubiesemos imaginado fruto de la situación pandémica actual y, en concreto, durante el período de confinamiento vivido por la crisis por Covid-19 han reavivado el debate sobre la necesidad de cambios tecnológicos en el proceso de enseñanza- aprendizaje.

La crisis provocada por la COVID-19 ha situado a la escuela en un escenario inédito. De un día para otro, la enseñanza -cuando esto ha sido posible- ha pasado de ser presencial a ... virtual. ... Profesores, alumnos y padres y madres están accediendo a contenidos didácticos ... que ponen de manifiesto que es posible aprender de otra manera, de un modo más autónomo. ... Es posible que, a partir de la vuelta a la docencia presencial, no sea preciso pasar tantas horas en la escuela y, muy especialmente, en el aula. (Feito, 2020, p.156)

O como señalan Fernández y Vergara (2020): Incluso antes del Covid-19 los cambios originados con la digitalización de la sociedad y las consecuencias de la implantación de la industria 4.0. estaban justificados en el sistema educativo por la responsabilidad de formar una futura ciudadanía con las habilidades técnicas y sociales necesarias para desarrollarse en un entorno cada vez más digitalizado. Estos cambios se están acelerando, precisamente, por la situación actual en la que la modalidad de enseñanza telemática implantada en España durante el confinamiento ha incrementado el uso de dispositivos digitales y herramientas virtuales que han permitido a estudiantes y docentes continuar con los procesos de enseñanza-aprendizaje desde casa.

\section{La fundamentación epistemológica del uso de las TIC digitales y su adecuación a los modelos pedagógicos vigentes}

En el apartado anterior se desarrolló la necesidad de la introducción y adecuación de las Tic, más que su introducción motivada por una adecuada necesidad. Aunque se podría llevar el cuestionamiento hasta el nivel teleológico del ¿para qué sirve la educación? Lo que es interesante, si se quiere, es también saber a quién sirve la educación: "Estará ella dirigida à formación exclusiva para los mercados, o deberá centrarse en una emergencia y afirmación de la persona?" (Reis, 2009).

Los paradigmas y enfoques educativos se conciben en función de varios factores entre los cuales están, sin duda, los instrumentos metodológicos y tecnológicos disponibles. Entre los modelos pedagógicos y los medios disponibles se entabló, desde siempre, una eterna contienda sobre su compatibilidad y la mejor combinación que garantice los más deseables horizontes educativos. 
http://doi.org/10.15359/ree.25-1.16

Uno de los grandes teóricos latinoamericanos, Kaplún (2001), influido por Freire, agrupó los modelos pedagógicos conforme a tres perspectivas pedagógicas: transmisora (de contenidos), persuasiva conductista (que moldea la conducta del alumnado y mide los efectos conseguidos) y problematizadora o cogestionaria. Esta última, precisamente, se plantearía que el estudiantado, a partir de ser capaz de pensar por sí mismo, tome conciencia de la realidad y contribuya a transformarla (véase apéndice A).

Cada uno de estos tres modelos parte de cómo son entendidos los conceptos esenciales en torno a qué es enseñar y qué es aprender; además de que identifica los roles que los actores (agentes) involucrados (personal educativo y estudiantes) desempeñan en el proceso de enseñanza/ aprendizaje. Un proceso que no puede sino tomarse como intrínsecamente comunicacional, aunque puedan diferir los roles de los sujetos involucrados y la naturaleza de los procesos.

Los paralelismos entre comunicación y educación que se trazan en el presente artículo tienen particular relevancia, no solo por que ambas son prácticas en las que se maneja información, conocimiento e, incluso, sabiduría destinada a una comunidad y construida por ella. Interesan, también y, sobre todo, porque la irrupción de las herramientas digitales en la sociedad ha trastocado el andamiaje conceptual y operativo tanto de una como de la otra.

Tal como señala Bernete (2010), los problemas que enfrentan hoy en día los estudios de la comunicación como los de la educación convergen en el modo en que la aplicación de las tecnologías más actuales, particularmente las digitales, afectan la transmisión de la información que llevan a cabo los agentes involucrados. Estos agentes son el sujeto emisor/sujeto receptor, en el caso de la comunicación y docente/discente, en el de la educación.

Sin embargo, queda una última consideración, pues existe una diferencia esencial entre información y conocimiento.

Mientras que la información representa meros datos, el conocimiento representa el significado de esos datos. [También señala que es el conocimiento el que] conlleva igualmente el poder para crear nuevos significados y estructuras, así como nuevas ideas y estrategias para aplicarlo en diferentes escenarios. (Arriaza Ibarra, 2005, p. 2)

Estos nuevos significados se materializan como nuevas ideas y estrategias, en escenarios que pueden ser muy diferentes de aquel en el que se originó el conocimiento de base. Los datos se referirían a símbolos de valores de variables cuantitativas o cualitativas sin un significado particular y, por lo tanto, sin añadir cualquier significado positivo, negativo o neutral al valor. Por su parte, la información representaría una respuesta a preguntas sobre el quién, qué, dónde y cuándo, se logran poner los datos en contexto, de modo que un sujeto mediante un comportamiento cognitivo produce una comprensión que hace efectiva una información, así transformada en útil. Ya el conocimiento involucra procesos cognitivos complejos que organizan la información dentro de un cuadro teórico, que tanto explica la realidad como puede suportar 
http://doi.org/10.15359/ree.25-1.16

http://www.una.ac.cr/educare

educare@una.ac.cr

intervenciones sobre ella con respuestas a preguntas sobre el cómo. La sabiduría sería un resultado cognitivo complejo que ultrapasa el qué, el cómo e, incluso, el por qué, propio de la comprensión, usándolos para definir lo que es mejor existencialmente.

Recuperando ahora la caracterización de los modelos pedagógicos señalados por Kaplún (2001), el modelo problematizador nos parece que sería el mejor para favorecer la emancipación, la autonomía, la cooperación y la creatividad de cara a la construcción de una sociedad justa. Si los modelos de Kaplún (2001) nos servirán para categorizar las posturas del profesorado, solo el modelo problematizador se tomará como preferencial y mejor garantía de la introducción de las TIC como una adecuada necesidad.

\section{Metodología}

Para llevar a cabo esta investigación y teniendo en cuenta el objetivo planteado, se propone una metodología cuantitativa, y como herramienta para llevarla a cabo hemos empleado un cuestionario aplicado a docentes de institutos de educación secundaria obligatoria (ESO) en Coimbra (Portugal).

\section{Objetivos}

Determinar el uso de las TIC digitales por parte del personal docente y su adecuación a los modelos pedagógicos vigentes.

\section{Sujetos y muestra}

Para contrastar el enfoque predominante del objeto de estudio presente en la bibliografía científica, se buscó establecer el perfil de conocer el uso que el personal docente hace en las aulas en la educación secundaria obligatoria (ESO) en Coímbra. La muestra fue aleatoria. En total han participado 100 docentes de institutos de la mencionada población portuguesa; a quienes, previamente, se les informó del objetivo que se pretendía en esta investigación. El rango de la edad de docentes que participaron se situaba entre los 40 y los 60 años, y la edad media de 55 años.

\section{Instrumentos}

Es importante destacar que el estudio tiene un carácter cuantitativo, por lo que se ha elaborado un cuestionario ad hoc compuesto de 14 preguntas para mostrar el uso y frecuencia de las nuevas tecnologías en el aula por parte del personal docente. Se dividió de acuerdo con las siguientes variables: Sexo, años de experiencia, uso de internet en el aula, horas que dedica el docente a 
http://doi.org/10.15359/ree.25-1.16

preparar las clases a través de internet y la frecuencia de uso de internet en las aulas. El estudio se enmarca en un diseño de corte cuantitativo, no experimental, descriptivo y correlacional.

El cuestionario fue validado por siete personas expertas del área de metodología de investigación. Antes de proceder a pasar el cuestionario se realizó una aplicación piloto a cuatro docentes para conocer si el cuestionario era comprensible.

Para la recogida de la información, los cuestionarios fueron distribuidos a través de los institutos, y se cumplimentaron en el tiempo de recreo. Una vez recopilada toda la información teórica que se consideró esencial para la investigación, y estructuradas y contextualizadas para la presente investigación, se procedió a analizar los datos obtenidos.

\section{Análisis de los resultados}

Para el análisis de los datos se han utilizado técnicas de corte cuantitativo, como señalábamos anteriormente. Teniendo en cuenta el carácter descriptivo de nuestro estudio, nos hemos centrado en el análisis de los resultados a través de medias, frecuencias y desviaciones estándar de los datos. Además, se analizaron los datos recogidos en los cuestionarios a través del paquete estadístico Statistical Package for the Social Sciences (versión 24.0 para Windows).

La distribución de la muestra respecto a la variable sexo ha estado compuesta por un $64,5 \%$ de mujeres y un 35,50 \% de hombres, tal y como, se puede apreciar en la Figura 1.

Figura 1: Distribución del sexo

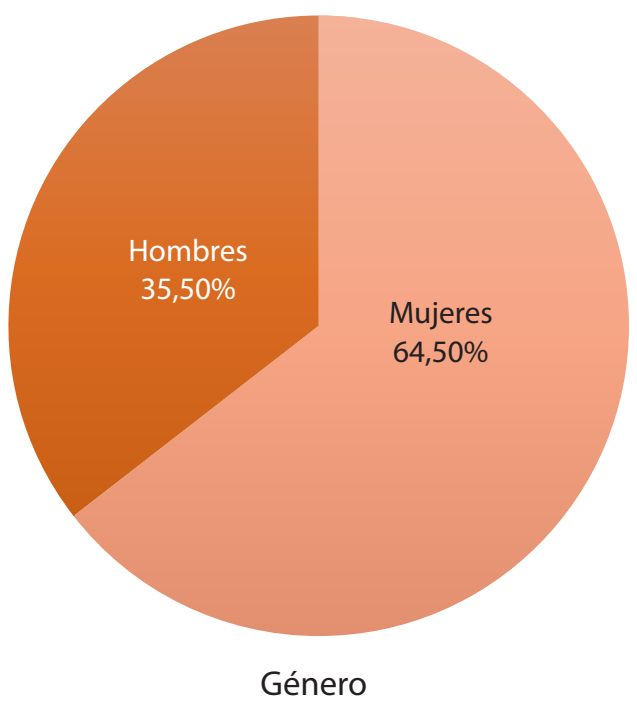

Nota: Elaboración propia. 
http://doi.org/10.15359/ree.25-1.16

http://www.una.ac.cr/educare

educare@una.ac.cr

Los resultados en relación con los años de experiencia en la universidad y el uso de las nuevas tecnologías que hace el personal docente en el aula, como queda reflejado en la Tabla 1, nos muestran que tan solo el $8 \%$ tiene experiencia entre 4 y 5 años como docente utilizando las tecnologías. El 22\% tiene una experiencia de 6 a 10 años, mientras que el porcentaje mayor se sitúa en un 29\% de docentes con una experiencia entre 11 y 20 años. Por último, los datos obtenidos nos muestran que el $41 \%$ del personal docente que lleva ejerciendo la docencia entre 21 a 30 años son quienes más usan las tecnologías en el aula y parecen con más predisposición a usarlas como instrumentos eficaces en el aula, puesto que las consideran fundamentales para incorporarlas en el proceso educativo. Además, parecen con más disposición a emplearlas como instrumentos eficaces en el aula y fundamentales para incorporarlas en el proceso educativo.

Tabla 1: Años de experiencia

\begin{tabular}{lccccc}
\hline & Frecuencia & Porcentaje & Porcentaje válido & Porcentaje acumulado \\
\hline Válido & $4-5$ & 8 & 8,0 & 8,0 & 8,0 \\
& 22 & 22,0 & 22,0 & 30,0 \\
& $6-10$ & 29 & 29,0 & 29,0 & 59,0 \\
& $11-20$ & 41 & 41,0 & 41,0 & 100 \\
& $21-30$ & 100,0 & 100,0 & \\
& Total & 100 & &
\end{tabular}

Nota: Elaboración propia.

En cuanto a si el personal docente usa internet en el aula comprobamos, tal y como se aprecia en la Tabla 2, que la mayoría del profesorado lo hace muy frecuente en un $83 \%$. Por el contrario, tan solo el $17 \%$ del personal docente solicita el uso de las TIC a sus estudiantes.

Los resultados reflejados en la Figura 2 sobre la frecuencia de uso de internet en las aulas que pide el personal docente a sus estudiantes muestran que la mayoría del profesorado lo hace muy frecuente en un $78 \%$. Por el contrario, tanto solo el $16 \%$ y el $6 \%$ del personal docente solicita el uso de las TIC a sus estudiantes alguna vez y nunca respectivamente. El 39\% del personal docente dedica de su tiempo usando internet para la preparación de las clases y presentaciones en estas más de 20 horas a la semana. Por su parte el porcentaje de docentes que dedican entre 13 a 20 horas preparando sus clases y haciendo presentaciones con el uso de las nuevas tecnologías es de un 19\%. El 16\% del personal docente utiliza internet para preparar sus clases y presentaciones con una duración de entre 8 a 12 horas a la semana y el 14\% utiliza internet de 4 a 7 horas. Por último, el porcentaje de docentes que utilizan internet menos de 3 horas para la preparación de sus clases y presentaciones representa el 12\%. 
Tabla 2: Uso de internet en el aula

\begin{tabular}{cccccc}
\hline & & Frecuencia & Porcentaje & Porcentaje válido & Porcentaje acumulado \\
\hline \multirow{2}{*}{ Válido } & Sí & 83 & 83,0 & 83,0 & 83,0 \\
& No & 17 & 17,0 & 17,0 & 100 \\
& Total & 100 & 100,0 & 100,0 & \\
\hline
\end{tabular}

Nota: Elaboración propia.

Del análisis de los datos sobre las horas que dedica el personal docente a preparar las clases a través de internet, los resultados aparecen en la Tabla 3 y la Figura 2.

Tabla 3: Horas dedicadas al uso de las nuevas tecnologías para preparación de las clases y presentación en estas mismas

\begin{tabular}{ccccc}
\hline & Frecuencia & Porcentaje & Porcentaje válido & Porcentaje acumulado \\
\hline Menos de 3 & 12 & 12,0 & 12,0 & 12,0 \\
4 a 7 & 14 & 14,0 & 14,0 & 26,0 \\
8 a 12 & 16 & 16,0 & 16,0 & 42,0 \\
13 a 20 & 19 & 19,0 & 19,0 & 61,0 \\
más de 20 & 39 & 39,0 & 39,0 & 100 \\
\hline Total & 100 & 100,0 & 100,0 & \\
\hline
\end{tabular}

Nota: Elaboración propia.

Figura 2: Frecuencia de preparación clases del personal docente y presentación con TIC digitales en el aula

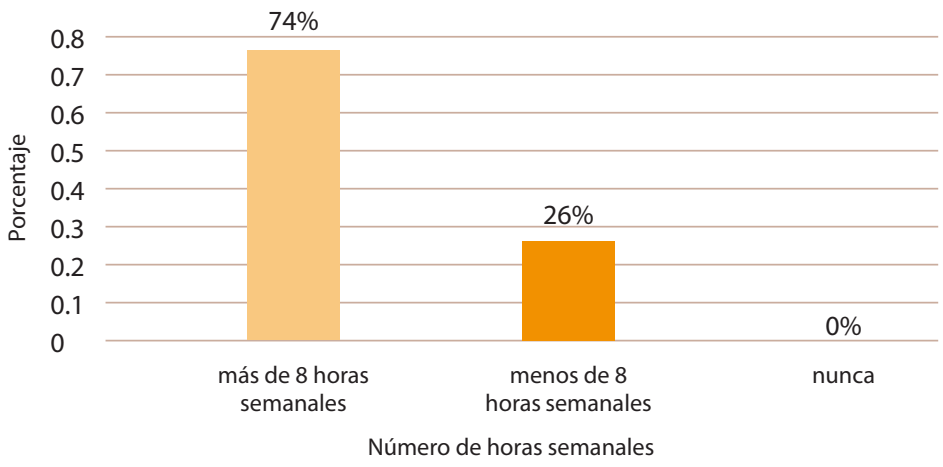

Nota: Elaboración propia. 
http://doi.org/10.15359/ree.25-1.16

http://www.una.ac.cr/educare

educare@una.ac.cr

Figura 3: Número de horas de uso de internet semanales

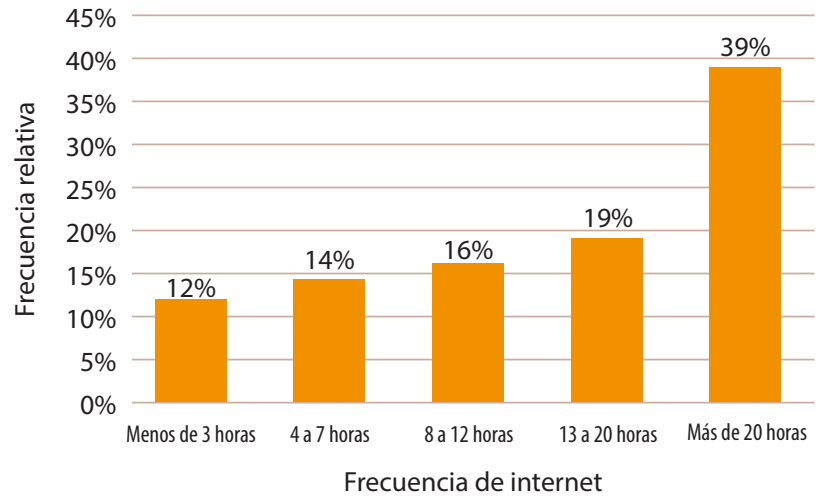

En la Figura 3 comprobamos que un 39\% del profesorado emplea más de 20 horas conectado a internet para preparar sus clases y tan solo un 12\% menos de 3 horas.

\section{Consideraciones finales}

De los resultados que se han obtenido en el estudio podemos destacar las siguientes conclusiones:

Es imprescindible considerar que, tras los elogios de los nuevos dispositivos, la estupefacción ante la avalancha de información disponible y los beneficios socio-económicos que estas tecnologías pueden alcanzar, se puede estar eludiendo un debate más profundo sobre los fundamentos epistemológicos implícitos en el uso de las nuevas herramientas digitales, respecto a si son una herramienta docente adecuada, acorde a los modelos educativos del presente.

La integración de las TIC en el aula ha sido un proceso que ha necesitado una alfabetización para todos los docentes y las docentes a lo largo de estos años. Por ello, consideramos que nuestra investigación ha cumplido los objetivos señalados, el uso de las TIC en las aulas, desarrollando un aprendizaje innovador y flexible.

A la vista de los resultados del estudio se podría deducir que el poco uso o nulo de las TIC en el aula venga motivado por la falta de competencia digital por parte del profesorado 
http://doi.org/10.15359/ree.25-1.16

El modelo unidireccional de formación, donde el saber recae sobre el personal docente ha quedado obsoleto. Se hace necesario innovar hacia modelos más flexibles en los que el personal docente actúe en calidad de guía para que sus estudiantes sean formados con más autonomía en su proceso de enseñanza-aprendizaje mediante el uso de las nuevas tecnologías. Las TIC han permitido trabajar la autonomía de cada estudiante, fomentando la participación activa en el aprendizaje; para ello, la alfabetización que ha tenido o tiene que aprender el personal docente permite facilitar la construcción de una nueva metodología en las aulas, en donde apliquen la comunicación y la información en todos los contextos sociales.

El personal docente destaca el uso de las TIC, de forma fácil y dinámica mejorando el desarrollo de enseñanza aprendizaje y a su vez favoreciendo el desarrollo de sus discentes. De modo que no cabe duda de que es fundamental la preparación en el uso de las TIC por parte del profesorado, sea una competencia básica en su labor educativa para que hagan uso de ellas en las aulas, en pro de una educación más flexible e innovadora cada día.

Además, el debate de la necesidad de que el personal docente esté actualizado en materia tecnológica implica un tiempo y coste que se hace cada día más necesario, teniendo en cuenta que el número de docentes que las emplean en su labor educativa, conforme a los resultados de nuestro estudio, es un alto porcentaje. No obstante, el profesorado que usa alguna vez o nunca la competencia digital en el aula puede deberse a que tengan miedo a utilizar las TIC por temor a que el alumnado conozca mejor la competencia digital.

El uso de internet en las aulas ha generado que el nuevo estudiantado tenga la posibilidad de tener mayor calidad de enseñanza mediante la integración de metodologías activas, flexibles y dinámicas que llevan a cabo su profesorado en las aulas, o lo que es lo mismo, gracias al cambio en los roles tradicionales del personal docente y el alumnado.

La innovación educativa gracias a las TIC ha ido sumergiéndose en las aulas como otra metodología nueva del proceso de enseñanza- aprendizaje. Esta va desde el acceso al conocimiento, hasta procesar la información usando entornos diferentes de comunicación en entornos de interacción social. Así, las demandas de las TIC en las aulas han provocado cambios en la metodología de la enseñanza que pueden constituir un instrumento didáctico que cada vez genera mayor interés dentro de las actividades docentes. De manera que cada día se hace más necesario que el personal docente sea consciente de la importancia de salir de la zona de confort, de la enseñanza meramente tradicional e incoporar las TIC en la educación en general y en la universitaria, en particular, de una forma más sistemática y regular. Ello requiere de un cambio metodológico en la manera en cómo el estudiantado aprende y el profesorado enseña.

Igualmente, otras de las conclusiones que se han extraído de los cuestionarios permite analizar nuevas variables, abriendo otras posibilidades a nuevos estudios de investigación entre 
http://doi.org/10.15359/ree.25-1.16

http://www.una.ac.cr/educare

educare@una.ac.cr

las competencias digitales del profesorado y del alumnado. De ese modo, una posible línea de continuidad en este estudio es la de comprobar, si el personal docente, además de usar las nuevas tecnologías en las aulas, lo hace para trabajar en equipo con otros compañeros y compañeras y con qué frecuencia lleva cursos de actualización y formación en TIC.

No olvidemos que las TIC conviven en el aula como estrategia educativa y que aunque con pros y contras en el aula destaca su potencial de ser una fuente de recursos educativos que permite un mayor contacto con el estudiantado, facilitando tareas más creativas, liberando al personal docente de los trabajos repetitivos y monótonos, y motivando la actualización profesional; pero, por el contrario, requiere de entusiasmo y subordinación a los sistemas informáticos ante los que no todo el personal docente está preparado. Y como señalan de Felipe y Briz (2004):

No todo son parabienes con las nuevas TIC y en el fenómeno de globalización, como todos sabemos, hay aspectos positivos y negativos que conviene analizar de forma objetiva, descartando la polémica que acompaña a muchas de estas discusiones. Los argumentos son muy variados y hay razones en las diferentes posturas, lo que encrespa el debate. (p.297)

En resumen, el debate sobre el uso de las tecnologías como estrategia didáctica en el aula está abierto a la reflexión, es dinámico y, sin duda, hay que seguir recorriendo un largo camino, seguir investigando en el ámbito de, si la repercusión tiempo-coste en términos de éxito académico de sus estudiantes es significativa o menos de lo esperado y cuáles estiman son fundamentales para usar en el aula en beneficio de un mejor rendimiento en sus estudiantes.

Además, la realidad educativa actual implica una renovación del sistema educativo, que se está quedando obsoleto, para hacer frente a unas necesidades socioeducativas latentes en el marco escolar actual, para que se adecue mejor a los nuevos retos a los que se ve sometida la sociedad. De hecho, entendemos que los estudios sobre el desarrollo de la competencia digital del personal docente tras el auge del uso de las nuevas tecnologías de la información y del conocimiento tras el confinamiento están y deben seguir proliferando. Pues, en este contexto de incertidumbre, provocado por una pandemia mundial, la digitalización, más que nunca, parece que ha venido para quedarse.

Igualmente, nos hacemos la siguiente reflexión sobre la falta de conocimiento en el ámbito de la enseñanza en línea, por parte de algunos docentes y algunas docentes sobre la necesidad de llevar a cabo cursos de formación tanto para docentes como para estudiantes. 


\section{Referencias}

Altamirano Herrera, S. A. y Lera Mejía, J. A. (2017). Futuro de las tic's para una educación incluyente. En J. A. Lera Mejia (Organizador), II Congreso sobre Desigualdad social, económica y educativa en el siglo XXI (pp. 416-428). Universidad Autónoma de Tamaulipas. http://www.eumed. net/libros-gratis/actas/2017/desigualdad/30-futuro-de-las-tics-para-una-educacionincluyente.pdf

Área, M. (2005). Tecnologías de la información y la comunicación en el sistema escolar. Una revisión de las líneas de investigación. Revista Electrónica de Investigación y Evaluación Educativa, 11(1), 3-25. https://doi.org/10.7203/relieve.11.1.4194

Area, M. y Guarro, A. (2012). La alfabetización informacional y digital: Fundamentos pedagógicos para la enseñanza y el aprendizaje competente. Revista Española de Documentación Científica, 35(Monográfico), 46-74. https://doi.org/10.3989/redc.2012.mono.977

Arriaza Ibarra, K. (2005). Las TIC en la teoría del conocimiento: Conocimiento e información. Área abierta, 12, 1-6. https://dialnet.unirioja.es/ejemplar/125078

Bernete, F. (2010). Usos de las TIC, relaciones sociales y cambios en la socialización de las y los jóvenes. Revista de Estudios de juventud, 88, 97-114. https://www.researchgate. net/publication/45413241_Usos de las TIC Relaciones sociales_y cambios en_la socializacion de las y los jovenes

Bernete, F. (2014). Cambios en la educación asociados a nuevas plataformas comunicativas. En J. F. Duran Medina y S. Durán Valero (Coords.), La era de las TIC en la nueva docencia (pp.7184). McGraw Hill. https://www.academia.edu/31918465/Libro 5 v3 La era de las TT II CC v?auto=download

Briggs, A. y Burke, P. (2002). De Gutenberg a internet: Una historia social de los medios de comunicación. Taurus.

Bustos, A. y Román, M. (2011). La importancia de evaluar la incorporación y el uso de las TIC en educación. Revista Iberoamericana de Evaluación Educativa, 4(2), 1-5. https://revistas.uam. es/riee/article/view/4452

Cabero Almenara, J. (2007). Nuevas tecnologías aplicadas a la educación. McGraw Hill. 
http://doi.org/10.15359/ree.25-1.16

http://www.una.ac.cr/educare

educare@una.ac.cr

Cairncross, F. (1997). The death of distance: How the communications revolution will change our lives. Harvard Business School Press.

Cornejo, M. y Tapia, M. L. (2011). Redes sociales y relaciones interpersonales en internet. Fundamentos en Humanidades, 12(24), 219-229. https://www.redalyc.org/articulo. oa?id=18426920010

Cruz, J. C. da (2002). Introdução ao estudo da comunicação: Imprensa, cinema, rádio, televisão, redes multimédia. Universidade Técnica de Lisboa, ISCSP.

de Felipe, I. y Briz, J. (2004). Cooperación y desarrollo ante las nuevas tecnologías en el marco del siglo XXI. CIRIEC-España, Revista de Economía Pública, Social y Cooperativa, 49, 295-319. http://ciriec-revistaeconomia.es/wp-content/uploads/12 De Felipe_y Briz 49.pdf

Díaz-Barriga, F. (2008). Educación y nuevas tecnologías de la información: ¿Hacia un paradigma educativo innovador? Sinectica. Revista electrónica de Educación, 30, 1-15. https://sinectica. iteso.mx/index.php/SINECTICA/article/view/192/185

Eshet-Alkalai, Y. (2004). Digital literacy: A conceptual framework for survival skills in the digital era. Journal of Educational Multimedia and Hypermedia, 13(1), 93-106. https://www. learntechlib.org/primary/p/4793/

Fainholc, B. (2011). Un análisis contemporáneo del Twitter. RED, Revista de Educación a Distancia, 26, 1-12. http://www.um.es/ead/red/26/fainhold.pdf

Feito, R. (2020). Este es el fin de la escuela tal y como la conocemos. Unas reflexiones en tiempo de confinamiento. Revista de Sociología de la Educación-RASE, 13(2), 156-163. http://dx.doi. org/10.7203/RASE.13.2.17130.

Fernández, P. y Vergara, D. (9 de junio, 2020). La digitalización del sistema educativo como consecuencia del Covid-19. Magisterio. https://www.magisnet.com/2020/06/ladigitalizacion-del-sistema-educativo-como-consecuencia-del-covid-19/

Fernández Enguita, M. (31 de marzo, 2020). Una pandemia imprevisible ha traído la brecha previsible. Cuaderno de campo [Mensaje en un blog]. https://blog.enguita.info/2020/03/ una-pandemia-imprevisible-ha-traido-la.html 
http://doi.org/10.15359/ree.25-1.16

http://www.una.ac.cr/educare educare@una.ac.cr

Flores-Tena, M. J. y Ortega-Navas, M. del C. (2018). El uso de las nuevas tecnologías: Una estrategia didáctica en los procesos de enseñanza-aprendizaje. Gedisa. https://www.researchgate.net/ publication/336020345 El uso de las nuevas tecnologias una estrategia didactica en los procesos de ensenanza-aprendizaje

Fujita, M., Krugman, P.y Venables, A. J. (1999). The spatial economy. Cities, regions and international trade. MIT Press. https://doi.org/10.7551/mitpress/6389.001.0001

García Aretio, L. (2009). ¿Por qué va ganando la educación a distancia? UNED.

García-Peñalvo, F. J. y Seoane-Pardo, A. M. (2015). Una revisión actualizada del concepto de eLearning. Décimo Aniversario. Education in the Knowledge Society, 16(1), 119-144. https:// doi.org/10.14201/eks2015161119144

Hodges, C., Moore, S., Lockee, B., Trust, T. y Bond, A. (27 de marzo, 2020). The difference between emergency remote teaching and online learning. Educause Review. https://bit.ly/3b0Nzx7

Kaplún, M. (2001). A la educación por la comunicación. La práctica de la comunicación educativa

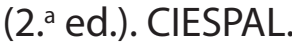

León Martínez, J. y Tapia Rangel, E. (2013). Educación con TIC para la sociedad del conocimiento. Revista Digital Universitaria, 14(1), 1-12. http://www.revista.unam.mx/vol.14/num2/ art16/\#up

Marqués Graells, P. (2013). Impacto de las TIC en la educación: Funciones y limitaciones. 3 c TIC: Cuadernos de desarrollo aplicados a las TIC, 2(1), 1-15. https://www.3ciencias.com/wpcontent/uploads/2013/01/impacto-de-las-tic.pdf

McCharen, B. Song, J. y Martens, J. (2011). School innovation:The mutual impacts of organizational learning and creativity. Educational Management Administration \& Leadership, 39(6), 676694. https://doi.org/10.1177/1741143211416387

Mcluhan, M. (2013). Understanding media. The extensions of man. MIT Pres.

Naciones Unidas, Comisión de Ciencia y Tecnología para el Desarrollo. (27 de febrero de 2014). Tecnologías de la información y las comunicaciones. Autor. https://unctad.org/meetings/ es/SessionalDocuments/ecn162014d3 es.pdf 
http://doi.org/10.15359/ree.25-1.16

http://www.una.ac.cr/educare

educare@una.ac.cr

Ortega Navas, M. del C. (2011). Las nuevas tecnologías como instrumentos innovadores de la educación a lo largo de la vida. Revista Española de Pedagogía, 69(249), 323-338. https:// revistadepedagogia.org//xix/no-249/las-nuevas-tecnologias-como-instrumentosinnovadores-de-la-educacion-a-lo-largo-de-la-vida/101400010194/

Quah, D.T. (1996). The invisible hand and the weightless economy (paper $\mathrm{N}^{\circ} 12$ ). Centre for Economic Performance. London School of Economics.

Reis, C. F. S. (2009). A educação face ao (des)aparecimento do sujeito. En A. Dujo, J. Boavida y V. Bento Guarda (Coords.), Educação: Reconfiguração das suas fronteiras (pp. 29-48). Centro de Estudos Ibéricos.

Tello Díaz Maroto, I. y Cascales Martínez, A. (2015). Las TIC y las necesidades específicas de apoyo educativo: Análisis de las competencias TIC en los docentes. RIED. Revista Iberoamericana de Educación a Distancia, 18(2), 355-383. https://doi.org/10.5944/ried.18.2.13536 


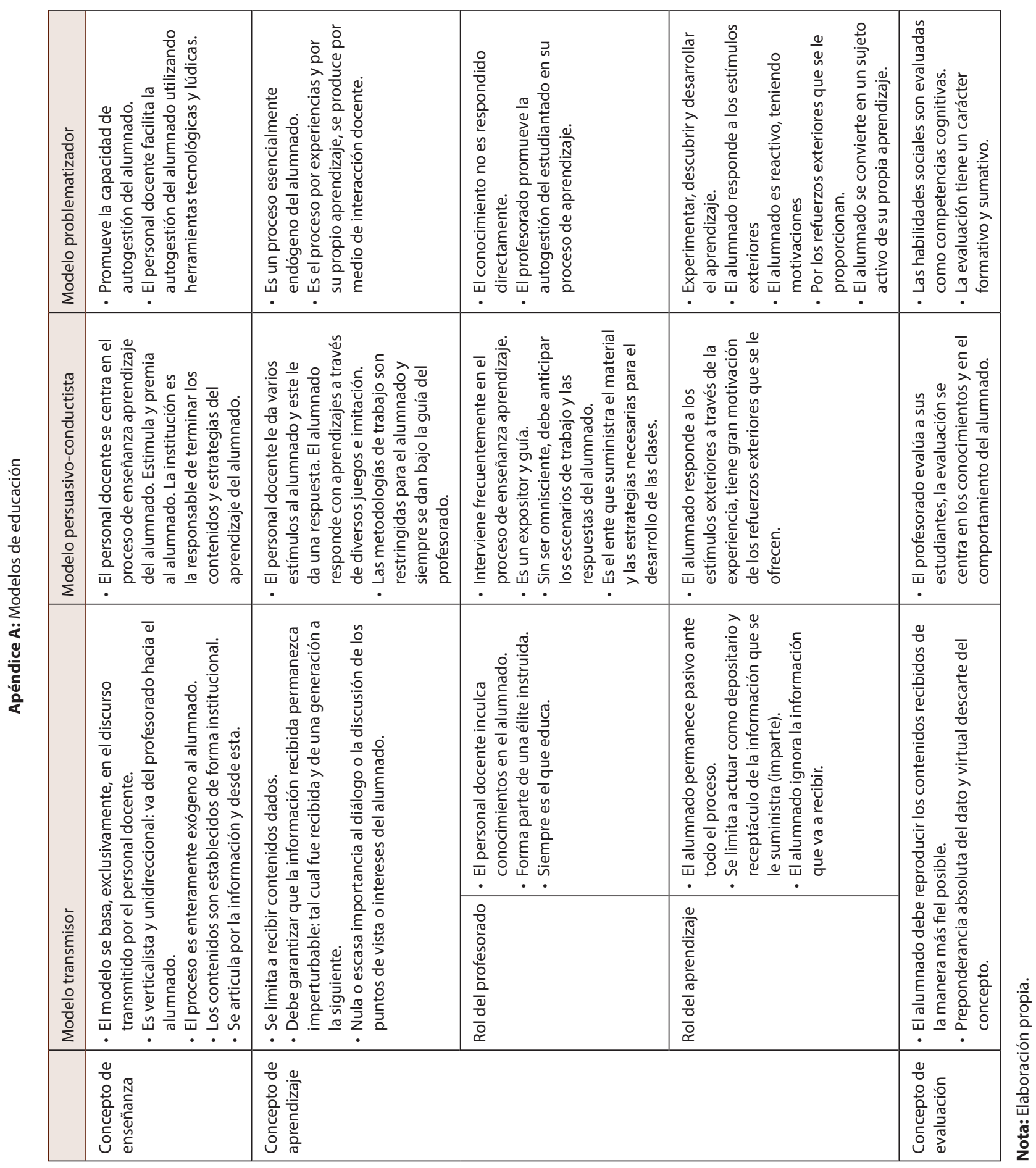

